\section{Short-term administration of JAK2 inhibitors reduces splenomegaly in mouse models of $\beta$-thalassemia intermedia and major}

$\beta$-thalassemia is one of the most common congenital red blood cell (RBC) disorders characterized by limited $\beta$ globin synthesis and ineffective erythropoiesis. ${ }^{1}$ Based on the requirements for blood transfusion, the disease can be classified as non-transfusion dependent thalassemia (NTDT), or transfusion dependent thalassemia (TDT). ${ }^{2}$ We previously demonstrated that in $\beta$-thalassemia, in response to anemia and hypoxia, high levels of erythropoietin (EPO) are associated with increased activation of the JAK2/STAT5 pathway and number of erythroid progenitors, especially in the spleen. ${ }^{3}$ The extramedullary hematopoiesis $(\mathrm{EMH})$ leads to hepatosplenomegaly which enhances the entrapment of $\mathrm{RBCs}$ in the spleen and exacerbates the anemia, further worsening the hepatosplenomegaly. Although splenectomy has now become almost obsolete in patients with TDT, the procedure is still more frequently used in patients with NTDT as it may improve anemia and avoid the need for transfusions. ${ }^{4}$ Unfortunately, this procedure remarkably increases the rate of thromboembolic complications. ${ }^{5}$

Clinical studies in patients affected by myeloproliferative disorders, characterized by activating JAK 2 mutations (such as polycythemia vera, PV), suggest that JAK2 inhibitors (JAK2i) are an effective treatment for splenomegaly. ${ }^{6-10}$ Given the central role of the EPO/JAK axis in $\beta$-thalassemia, we hypothesized that targeting JAK2 could be effective in reversing splenomegaly, and therefore could be used as an alternative to splenectomy. In fact, preliminary studies showed that the use of a JAK2i improved splenomegaly in a mouse model of $\beta$-thalassemia intermedia or NTDT $\left(H b b^{t / 3 /+}\right){ }^{3}$ In this subsequent study, we administered the JAK2i INCB018424 (Ruxolitinib) and TG101348 (Fedratinib, SAR302503) for 10 days to $H b b^{t h 3 /+}$ mice, and to mice requiring chronic blood transfusions for survival (TDT). TDT mice (C57FLC $\left.^{\text {th3/th3 }}\right)$ were generated by transplanting fetal liver cells

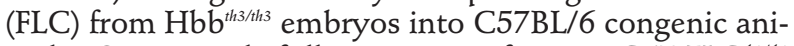
mals. One month following engraftment, C57-FLC ${ }^{\text {th } 3 \text { th } 3}$ mice exhibited prominent splenomegaly and severe ineffective erythropoiesis, rapidly becoming dependent on chronic blood transfusion for survival. ${ }^{11} \mathrm{Hbb}^{\text {th } 3 / 4}$ mice treated with either JAK2i showed a mild reduction in hemoglobin $(\mathrm{Hb})$ levels, $(8-10 \%)$, and RBC (8-9\%) when compared to vehicle-treated animals (Figure $1 \mathrm{~A}$ and $\mathrm{B}$ ). This mild worsening of anemia, however, was sufficient to increase serum EPO levels compared to placebo-treated $\mathrm{Hbb}^{\text {th3/+ }}$ controls (up to $84 \%$ ) (Figure 1C). Both JAK2i significantly reduced spleen weight $(60 \%$ for INCB018424 and 31\% for TG101348; Figure 1D) and reticulocyte numbers (Online Supplementary Figure S1A). Compared to placebo-treated mice, flow cytometry studies revealed that animals receiving JAK2i exhibited a reduction in the number of erythroid nucleated cells in the spleen but not in the bone marrow (BM) (Figure 1E and Online Supplementary Figure $S 1 B) .{ }^{12}$ This suggests that JAK2i might target preferentially rapidly proliferating erythroid progenitors in the spleen, as in extramedullary stress erythropoiesis. No differences were observed in serum HEPCIDIN levels (Online Supplementary figure 2A) or other iron parameters (Online Supplementary Figure $S 2 B-F)$. Since the reduction in splenomegaly was associated with mild anemia, we investigated whether coadministration of a JAK2 $\mathrm{i}$ with transfusion could further reduce the splenomegaly whilst preventing the anemia. We first tested this hypothesis by transfusing RBCs from transgenic animals expressing GFP into NTDT $H_{b b^{t h /+}}$ mice. GFP animals were utilized in order to discriminate by flow cytometry the relative contribution of endogenous versus donor RBCs in circulation at the end of treatment. Weekly transfusions started one week prior to initiating treatment with JAK2i and continued throughout the experiment (for a total of three weeks). As expected, blood transfusions reduced splenomegaly (28\%) compared to non-transfused controls (Figure 2A). When the transfusion was combined with the administration of JAK2 $\mathrm{i}$ for 10 days, the spleen weight was further reduced by $64 \%$ for INCB018424 and 45\% for TG101348 versus animals that received transfusion alone (Figure 2A). This observation was supported by flow cytometry studies, which showed reduction in the proportion of erythroid nucleated cells in the spleen (Figure 2B and Online Supplementary Figure $S 3 A$ ). We did not observe any difference in $\mathrm{Hb}$ levels between transfused animals in presence or absence of JAK $2 \mathrm{i}$, although a trend towards lower $\mathrm{Hb}$ levels was noted in mice treated with both transfusion and JAK2i (Online Supplementary Figure S3B). The contribution of endogenous GFP-RBCs to the total number of RBCs was smaller in JAK2i treated mice compared to the placebo treated group, although the total number of transfused GFP+ RBCs did not significantly differ among groups (Figure 2C).

This finding, together with the reduction of nucleated erythroid cells in the spleen (Figure 1E, 2B, Online Supplementary Figure $S 1 A$ and $3 A$ ), indicates that the combination of JAK $2 \mathrm{i}$ and transfusion further suppresses extramedullary erythropoiesis in the spleen. However, in this setting, the loss of endogenous RBC was only partially balanced by the transfused RBCs (Figure 2C).

We then tested the JAK2i in TDT C57-FLCth3/th3 mice. Animals that received transfusions showed $35 \%$ reduction in spleen weight when compared with nontransfused animals (Figure 2D). The combination of blood transfusion with administration of JAK2i for 10 days further reduced spleen weight (84\% for INCB018424 and 79\% for TG101348; Figure 2D) when compared with untransfused animals, with neither detrimental nor incremental effects on $\mathrm{Hb}$ levels and number of RBCs in circulation (Online Supplementary Figure S3C and $D$ ).

Our first observation is that combined administration of JAK2i and transfusion can further reduce the splenomegaly. This would be very helpful in patients who are unable to receive transfusion and iron-chelation therapy as well as those with clinically symptomatic splenomegaly or hypersplenism. ${ }^{13}$ Based on our data, we can only speculate that this reduction may prevent exacerbation of the anemia in the long term. However, our data also indicate that this combination further suppressed endogenous erythropoiesis and RBC production. For this reason, potential clinical trials using these inhibitors should aim for a reduction of splenomegaly, rather than increasing the levels of total RBCs (endogenous + donor) in circulation.

Our second observation pertains to safety. Long-term use of JAK $2 \mathrm{i}$ has proven to be effective for the treatment of myeloproliferative disorders such as Polycythemia Vera. ${ }^{10,14}$ However, in these studies, the positive effect on splenomegaly was associated with thrombocytopenia due to the inhibitory effect of JAK2i on megakaryopoiesis. ${ }^{15}$ In this study, we administered two JAK2i for only 10 days and noted that the spleen weight was almost normalized when transfusion was combined with 
the JAK2i. This short treatment was not associated with thrombocytopenia (not shown), though could be a possibility if duration of treatment were prolonged.

Our third major observation is related to the variation in the reduction of splenomegaly comparing INCB018424 vs. TG101348, in absence or presence of transfusion (Figure 1D and 2A). These differences could be due to the distinct activity of the two compounds. INCB018424 is a selective JAK1/JAK2 inhibitor (respectively with IC50 $3.3 \mathrm{nM}$ and IC50 $2.8 \mathrm{nM}$ ) whilst TG101348 is a potent inhibitor of JAK2 (IC50 $3 \mathrm{nM}$ ) with 30 -fold less selectivity for JAK1. 10,14 Our data showed that the amelioration of splenomegaly observed using TG101348 is not as effective as INCB018424, suggesting
$H b b^{\text {th } 3 /+}$

A
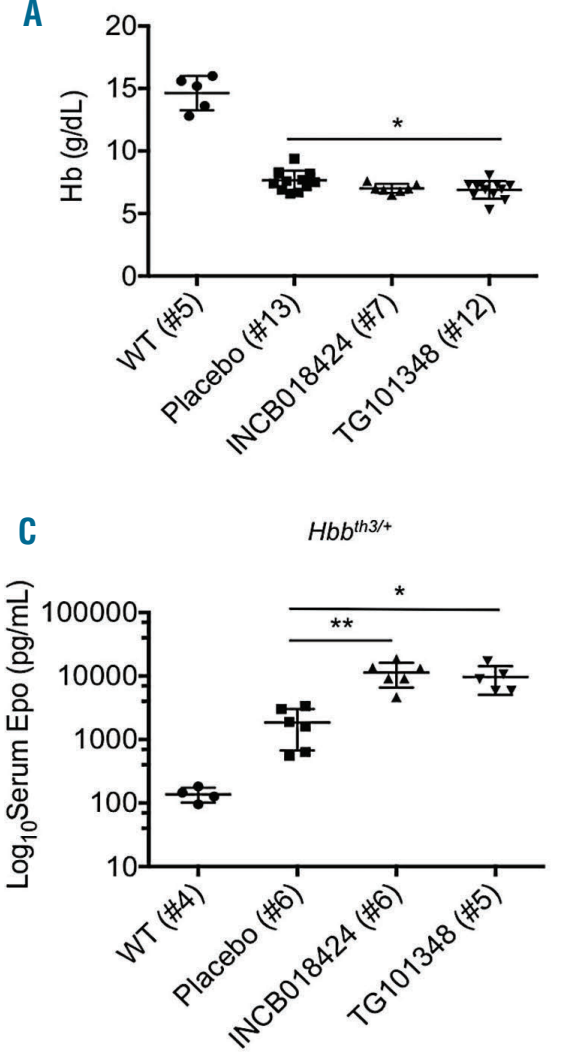

$H b b^{t h 3 /+}$

B

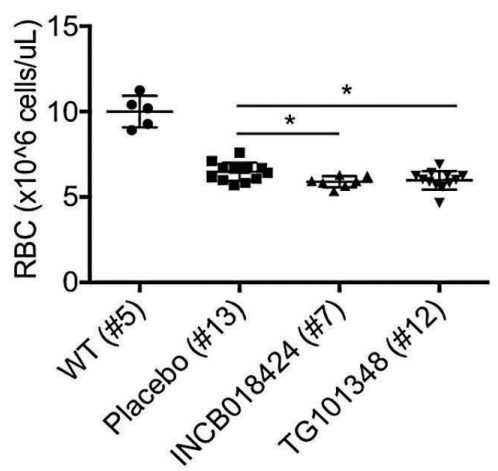

D

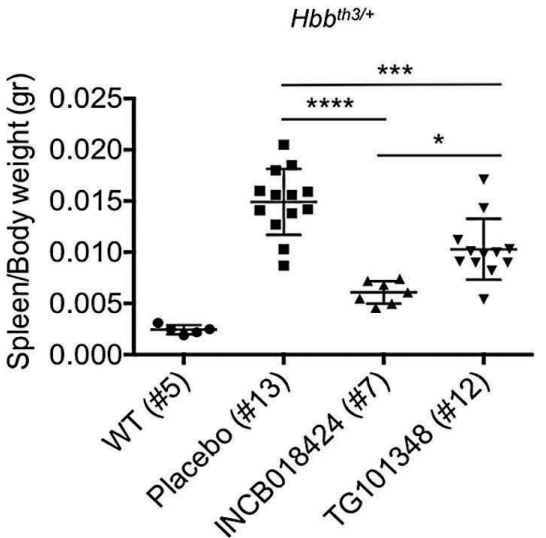

E

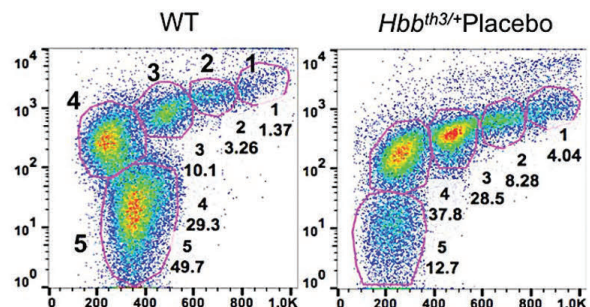

$\mathrm{Hbb}^{\text {th } 3 /+}$ INCB018424

$H b b^{\text {th } 3 /+} \mathrm{TG} 101348$
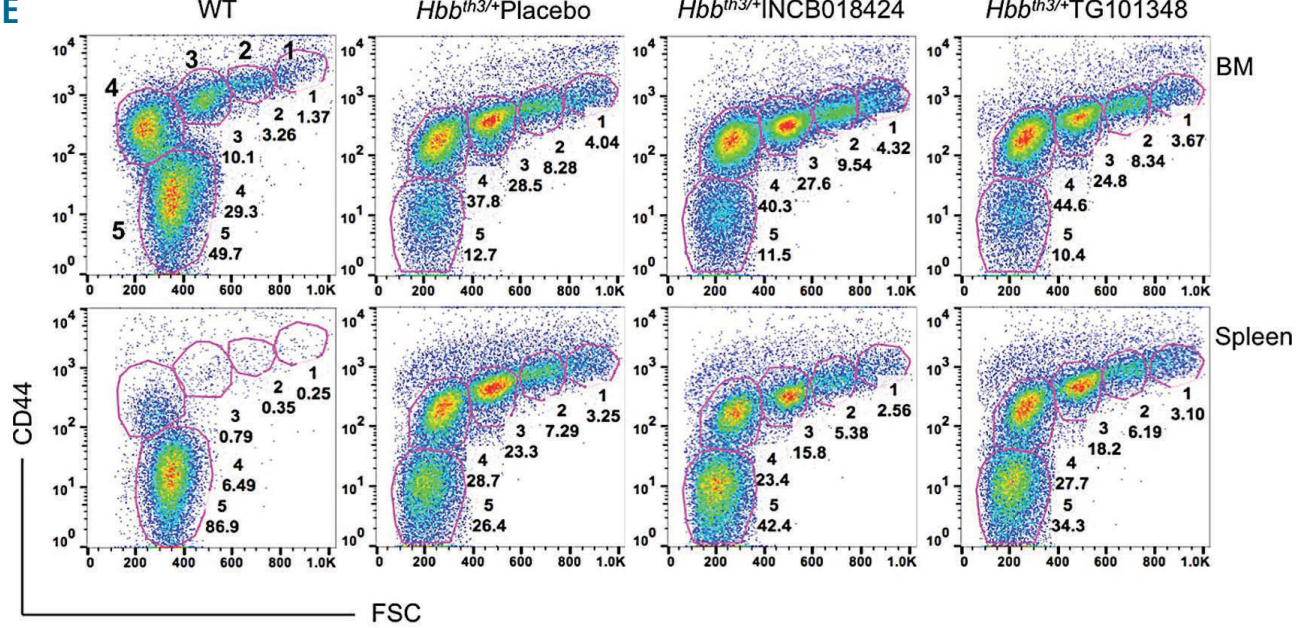

Figure 1. JAK2i significantly improved splenomegaly in a mouse model of $\beta$-thalassemia intermedia. Short-term treatment with the JAK2i INCB018424 or TG101348 in a mouse model of $\beta$-thalassemia intermedia (NTDT- $H b b^{\text {th }} 3 /+$ ) resulted in very mild exacerbation of anemia (A) and reduction in RBC number (B). This effect was associated with expected increase in serum EPO level (C). The significant reduction in splenomegaly suggests that JAK2i may target preferentially rapidly proliferating erythroid progenitors in the spleen, limiting their expansion and thus allowing the reduction in the spleen size (D). Some expected variability in spleen size was observed in control animals receiving the placebo which can be attributed to the diversity among mice. This variability seemed to be blunted by the administration of the inhibitors. Representative flow cytometry analysis (showing the different erythroid populations, in wild type (WT) and thalassemic animalsz, indicated the reduction of nucleated erythroid cells in the spleen (depicted by number 1 through 4; while population 5 represents RBC) after treatment with JAK2i, but not in the bone marrow (BM). (E). Analysis was performed using One-way ANOVA with Tukey multiple comparison adjustment. Results represent mean $\pm \mathrm{SD}, * * * * P<0.0001, * * * P<0.001, * * P<0.01, * P<0.05$. 
$H b b^{\text {th } 3 /+}$

A

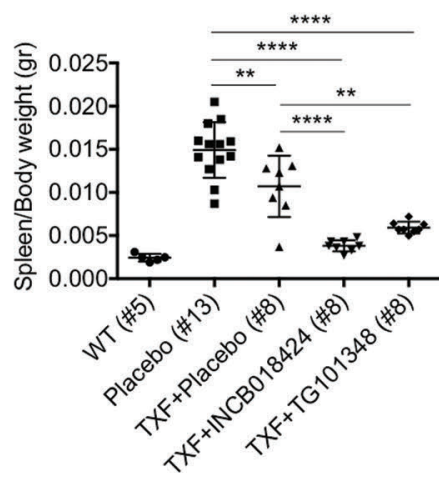

$H b b^{\text {th } 3 /+}$

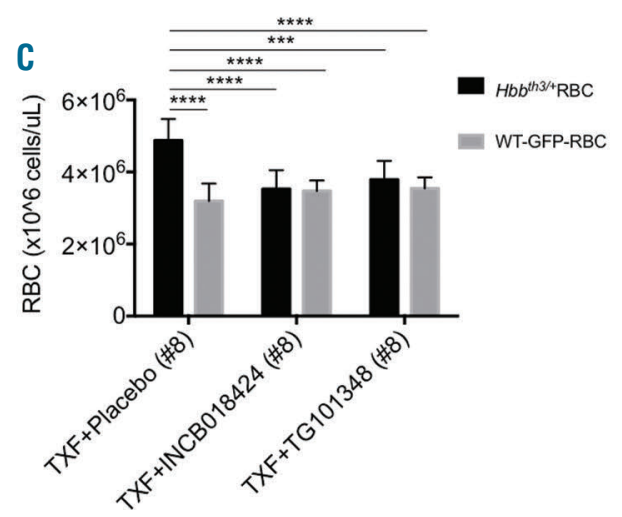

B
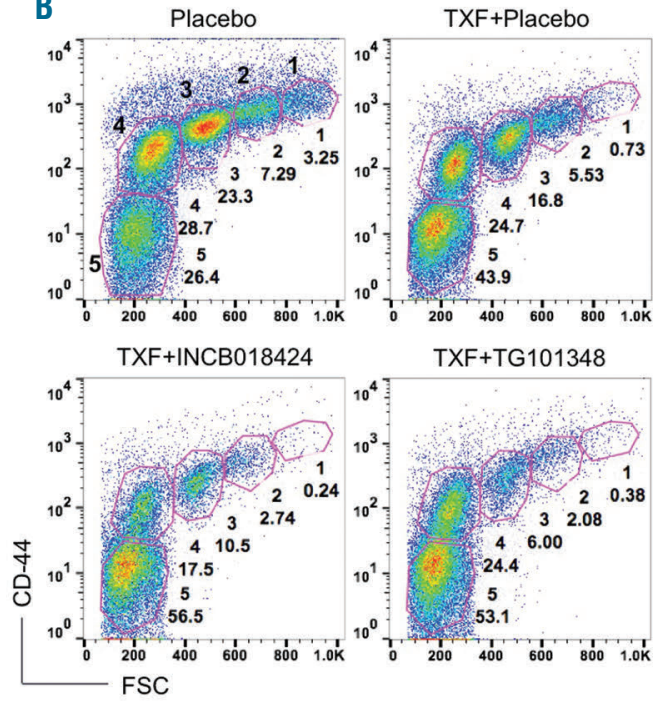

C57-FLC $C^{\text {th } 3 / \text { th } 3}$

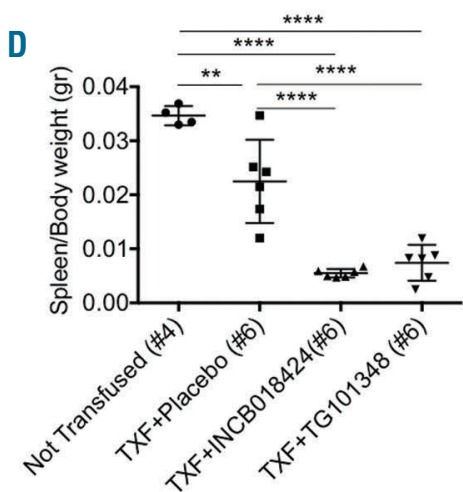

Figure 2. Co-administration of JAK2i and transfusion further improved extramedullary hematopoiesis. Administration of transfusion (indicated with TXF in the graphs) and JAK2 $i$ in a mouse model of $\beta$-thalassemia intermedia (NTDT$H_{b} b^{\text {th } 3 /+}$ ) almost normalized spleen size compared to wild type (WT) values (A). In the same mice, a reduction in the proportion of nucleated erythroid cells in the spleen was detected by flow cytometry (B). When the administration of a JAK2i was combined with transfusion, suppression of erythropoiesis was more pronounced, as indicated by a decreased number of endogenous RBC (C). Splenomegaly was also improved in a mouse model of $\beta$ thalassemia major (TDT-C57FLC $^{\text {th } 3 / \text { th } 3}$ ) when JAK2i and transfusion were administered simultaneously (D). Analysis was performed using One-way ANOVA with Tukey multiple comparison adjustment or Two-way ANOVA with Sidak's multiple comparison adjustment. Results represent mean \pm SD, $* * * * P<0.0001, \quad * * * P<0.001$, $\star * P<0.01$. a role of JAK1 in modulating extramedullary hematopoiesis and ineffective erythropoiesis. Further studies need to be performed in order to underline the role of Jak 1 in the pathophysiology of $\beta$-thalassemia.

In conclusion, we showed that short-term suppression of JAK2, and eventually also JAK1, in presence of transfusion can effectively reduce splenomegaly by limiting the number of nucleated erythroid cells with no detrimental effect on other lineages or anemia. This approach could be utilized as an alternative to splenectomy in thalassemic patients.

Carla Casu, ${ }^{1}$ Vania Lo Presti, ${ }^{1}$ Paraskevi Rea Oikonomidou, 1,2 Luca Melchiori, ${ }^{3}$ Osheiza Abdulmalik,' Pedro Ramos and Stefano Rivella

'Department of Pediatrics, Division of Hematology, Children's Hospital of Philadelphia (CHOP), PA, USA; ${ }^{2} P$ enteli's Children's Hospital, Pediatric Department, Athens, Greece; ${ }^{3}$ Adaptimmune Therapeutics Ltd., Abingdon, UK and ${ }^{4}$ Novartis Pharma AG, Basel, Switzerland

Correspondence: rivellas@email.chop.edu. doi:10.3324/haematol.2017.181511

Acknowledgments: mouse $C B C$ analysis was performed by the Translational Core Lab of the CHOP Research Institute.
Information on authorship, contributions, and financial \& other disclosures was provided by the authors and is available with the online version of this article at www. haematologica.org.

\section{References}

1. Rivella $S$. The role of ineffective erythropoiesis in non-transfusiondependent thalassemia. Blood Rev. 2012;26 Suppl 1:S12-15.

2. Weatherall DJ. Pathophysiology of thalassaemia. Baillieres Clin Haematol. 1998;11(1):127-146.

3. Libani IV, Guy EC, Melchiori L, et al. Decreased differentiation of erythroid cells exacerbates ineffective erythropoiesis in beta-thalassemia. Blood. 2008;112(3):875-885.

4. Musallam KM, Taher AT, Rachmilewitz EA. beta-thalassemia intermedia: a clinical perspective. Cold Spring Harb Perspect Med. 2012;2(7):a013482.

5. Crary SE, Buchanan GR. Vascular complications after splenectomy for hematologic disorders. Blood. 2009;114(14):2861-2868.

6. Jamieson C, Hasserjian R, Gotlib, et al. Effect of treatment with a JAK2-selective inhibitor, fedratinib, on bone marrow fibrosis in patients with myelofibrosis. J Transl Med. 2015;13:294.

7. Mesa RA. Ruxolitinib, a selective JAK1 and JAK2 inhibitor for the treatment of myeloproliferative neoplasms and psoriasis. IDrugs. 2010;13(6):394-403

8. Pasquier F, Cabagnols X, Secardin L, Plo I, Vainchenker W. Myeloproliferative neoplasms: JAK2 signaling pathway as a central 
target for therapy. Clin Lymphoma Myeloma Leuk. 2014;14 Suppl:S23-35.

9. Verstovsek S, Kantarjian H, Mesa RA, et al. Safety and efficacy of INCB018424, a JAK1 and JAK2 inhibitor, in myelofibrosis. N Engl J Med. 2010;363(12):1117-1127.

10. Wernig G, Kharas MG, Okabe R, et al. Efficacy of TG101348, a selective JAK2 inhibitor, in treatment of a murine model of JAK2V617Finduced polycythemia vera. Cancer Cell. 2008;13(4):311-320.

11. Rivella S, May C, Chadburn A, Riviere I, Sadelain M. A novel murine model of Cooley anemia and its rescue by lentiviral-mediated human beta-globin gene transfer. Blood. 2003;101(8):2932-2939.

12. Casu C, Oikonomidou PR, Chen $\mathrm{H}$, et al. Minihepcidin peptides as disease modifiers in mice affected by beta-thalassemia and poly- cythemia vera. Blood. 2016;128(2):265-276.

13. Taher A, Vichinsky E, Musallam K, Cappellini MD, Viprakasit V. In: Weatherall $\mathrm{D}$, ed. Guidelines for the management of non transfusion dependent thalassaemia (NTDT). Nicosia, Cyprus, 2013.

14. Quintas-Cardama A, Vaddi K, Liu P, et al. Preclinical characterization of the selective JAK1/2 inhibitor INCB018424: therapeutic implications for the treatment of myeloproliferative neoplasms. Blood. 2010; 115(15):3109-3117.

15. Al-Ali HK, Stalbovskaya V, Gopalakrishna P, Perez-Ronco J, Foltz L. Impact of ruxolitinib treatment on the hemoglobin dynamics and the negative prognosis of anemia in patients with myelofibrosis. Leuk Lymphoma. 2016;57(10):2464-2467. 